University of Nebraska - Lincoln

DigitalCommons@University of Nebraska - Lincoln

August 2000

\title{
Search for antiproton decay at the Fermilab Antiproton Accumulator
}

S. Geer

Fermi National Accelerator Laboratory, Batavia, Illinois

J. Marriner

Fermi National Accelerator Laboratory, Batavia, Illinois

M. Martins

Fermi National Accelerator Laboratory, Batavia, Illinois

R. E. Ray

Fermi National Accelerator Laboratory, Batavia, Illinois

J. Streets

Fermi National Accelerator Laboratory, Batavia, Illinois

See next page for additional authors

Follow this and additional works at: https://digitalcommons.unl.edu/physicssnow

Part of the Physics Commons

Geer, S.; Marriner, J.; Martins, M.; Ray, R. E.; Streets, J.; and Wester, W., "Search for antiproton decay at the Fermilab Antiproton Accumulator" (2000). Gregory Snow Publications. 34.

https://digitalcommons.unl.edu/physicssnow/34

This Article is brought to you for free and open access by the Research Papers in Physics and Astronomy at DigitalCommons@University of Nebraska - Lincoln. It has been accepted for inclusion in Gregory Snow Publications by an authorized administrator of DigitalCommons@University of Nebraska - Lincoln. 


\section{Authors}

S. Geer, J. Marriner, M. Martins, R. E. Ray, J. Streets, and W. Wester 


\title{
Search for antiproton decay at the Fermilab Antiproton Accumulator
}

\author{
S. Geer, J. Marriner, M. Martens, R. E. Ray, J. Streets, and W. Wester \\ Fermi National Accelerator Laboratory, Batavia, Illinois 60510 \\ M. Hu and G. R. Snow \\ University of Nebraska, Lincoln, Nebraska 68588 \\ T. Armstrong \\ Pennsylvania State University, University Park, Pennsylvania 16802 \\ C. Buchanan, B. Corbin, M. Lindgren, and T. Muller* \\ University of California at Los Angeles, Los Angeles, California 90024 \\ R. Gustafson \\ University of Michigan, Ann Arbor, Michigan 48109
}

(The APEX Collaboration)

(Received 10 August 1999; published 9 August 2000)

\begin{abstract}
A search for antiproton decay has been made at the Fermilab Antiproton Accumulator. Limits are placed on 13 antiproton decay modes. The results include the first explicit experimental limits on the muonic decay modes of the antiproton, and the first limits on the decay modes $e^{-} \gamma \gamma$ and $e^{-} \omega$. The most stringent limit is for the decay mode $\bar{p} \rightarrow e^{-} \gamma$. At $90 \%$ C.L. we find that $\tau_{p}^{-} / B\left(\bar{p} \rightarrow e^{-} \gamma\right)>7 \times 10^{5}$ yr. The most stringent limit for decay modes with a muon in the final state is for the decay $\bar{p} \rightarrow \mu^{-} \gamma$. At $90 \%$ C.L. we find that $\tau_{p}^{-} / B(\bar{p}$ $\left.\rightarrow \mu^{-} \gamma\right)>5 \times 10^{4}$ yr.

PACS number(s): 13.30.Ce, 11.30.Er, 11.30.Fs, 14.20.Dh
\end{abstract}

\section{INTRODUCTION}

The $C P T$ theorem requires that the proton and antiproton $(\bar{p})$ lifetimes are equal. Searches for proton decay have yielded lower limits on the proton lifetime $\tau_{p}>O\left(10^{32}\right) \mathrm{yr}$ [1]. Hence, a search for $\bar{p}$ decay with a short lifetime $\left(\tau_{p}^{-}\right.$ $\ll \tau_{p}$ ) tests the CPT theorem. In this paper we describe a search for $\bar{p}$ decay at the Fermilab Antiproton Accumulator.

$C P T$ invariance is one of the most fundamental symmetries of modern physics. Over the years there have been a variety of searches [1-4] for $C P T$ violation based on comparing particle and antiparticle masses, lifetimes, and magnetic moments. For example, the proton and antiproton masses have been shown to be equal with a precision of a few parts in $10^{8}$ [3], while the particle and antiparticle masses in the neutral kaon system have been shown to be equal to about one part in $10^{18}[1,4]$. A search for $\bar{p}$ decay complements these CPT tests by providing a search for $C P T$ violation accompanied by a violation of baryon number. Indeed, since the antiproton is the only long-lived antiparticle that could in principle decay into other known particles without violating charge conservation, a search for $\bar{p}$ decay provides a unique test of the $C P T$ theorem, and a unique test of the intrinsic stability of antimatter.

The sensitivity of a $\bar{p}$ decay search to the presence of a

\footnotetext{
*Present address: Universität Karlsruhe, 76128 Karlsruhe, Ger-
} many.
$C P T$ violating interaction has been characterized [5] by considering a dimension-6 $C P T$-violating operator with characteristic mass scale $m_{X}$. Dimensional analysis then provides the estimate $\tau_{p}^{-} \sim m_{X}^{4} / m_{p}^{5}$, yielding

$$
m_{X} \sim\left(4.3 \times 10^{9} \mathrm{GeV}\right) \times\left(\tau_{p}^{-} / 10 \mathrm{Myr}\right)^{1 / 4} .
$$

Hence, a search for $\bar{p}$ decay with a lifetime of order $1 \mathrm{yr}$, for example, provides a test for $C P T$ violation at a mass scale $\mathrm{O}\left(10^{7}\right) \mathrm{GeV} / c^{2}$, which is well beyond the scale accessible at high energy colliders.

The most stringent lower limit on $\tau_{p}^{-}$has been obtained [5] from a comparison of recent measurements of the cosmic ray $\bar{p}$ flux [7] with predictions based on expectations for secondary production of antiprotons in the interstellar medium. The agreement between the observed and predicted rates implies that $\tau_{p}^{-}$is not small compared to $T / \gamma$, where $T$ is the $\bar{p}$ confinement time within the galaxy $\left(\sim 10^{7} \mathrm{yr}\right)$ and $\gamma$ is the Lorentz factor for the observed antiprotons. After taking into account the uncertainties on the relationship between the interstellar $\bar{p}$ flux and the flux observed at the Earth, at $90 \%$ C.L. the limit $\tau_{p}^{-}>8 \times 10^{5}$ yr has been reported [5]. This indirect limit is not valid if current models of $\bar{p}$ production, propagation, and interaction in the interstellar medium are seriously flawed. Indeed, it has been claimed [6] that, within the framework of the minimal supersymmetric extension to the standard model, if neutralino annihilations contribute significantly to the flux of cosmic ray antiprotons 


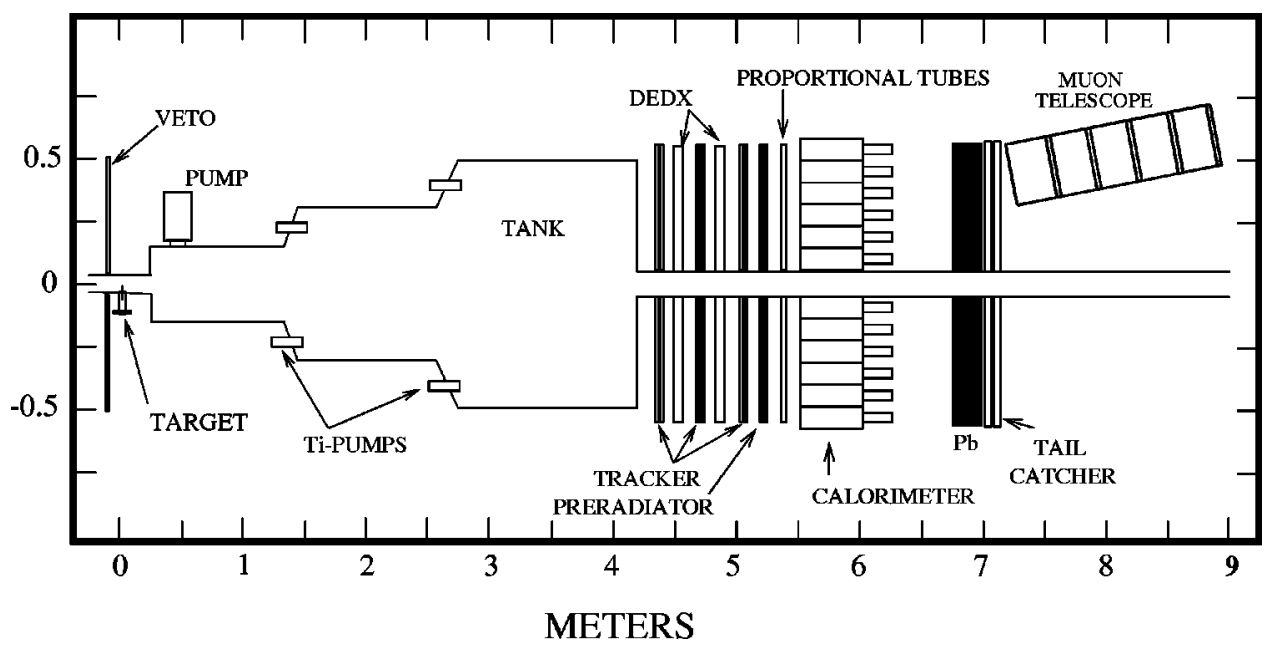

FIG. 1. Schematic of the APEX detector.

in the galactic halo, $\bar{p}$ lifetimes as low as $10^{5}$ years can accommodate the current cosmic ray data.

Laboratory searches for $\bar{p}$ decay have, to date, provided less stringent limits on $\tau_{p}^{-}$. However, these limits do not suffer from large model dependent uncertainties. The most stringent published laboratory limit on inclusive $\bar{p}$ decay has been obtained from a measurement of the containment lifetime of $\sim 1000$ antiprotons stored in an ion trap, yielding $\tau_{p}^{-}>3.4$ months [3]. The sensitivity of laboratory $\bar{p}$ decay searches can be dramatically improved by looking for explicit $\bar{p}$ decay modes at an antiproton storage ring. Angular momentum conservation requires that a decaying $\bar{p}$ would produce a fermion (electron, muon, or neutrino) in the final state. Hence, there are only a limited number of potential decay modes. A search for explicit $\bar{p}$ decay modes with an electron in the final state was made by the APEX test experiment (T861) at the Fermilab Antiproton Accumulator. The T861 search yielded the 95\% C.L. limits [8]: $\tau_{p}^{-} / B\left(\bar{p} \rightarrow e^{-} \gamma\right)>1848 \mathrm{yr}, \tau_{p}^{-} / B\left(\bar{p} \rightarrow e^{-} \pi^{0}\right)>554 \mathrm{yr}$, $\tau_{p}^{-} / B\left(\bar{p} \rightarrow e^{-} \eta\right)>171 \mathrm{yr}, \quad \tau_{p}^{-} / B\left(\bar{p} \rightarrow e^{-} K_{S}^{0}\right)>29 \mathrm{yr}, \quad$ and $\tau_{p}^{-} / B\left(\bar{p} \rightarrow e^{-} K_{L}^{0}\right)>9 \quad \mathrm{yr}$.

Following the T861 test experiment, the APEX detector $[9,10]$ was designed to enable a more sensitive search for $\bar{p}$ decay at the Fermilab Antiproton Accumulator. Based on APEX data, we have recently reported limits on $\tau_{p}^{-} / B$ from the first search for antiproton decay modes with a muon in the final state $[12,13]$. In this paper we give a more detailed description of our limits on $\bar{p}$ decay modes with a muon in the final state, and describe new results from a search for $\bar{p}$ decay modes with an electron in the final state.

The paper is organized as follows. Section II gives a brief description of the APEX detector. The APEX triggers and data samples are described in Sec. III. The search for decay modes with a muon in the final state is described in Sec. IV, and the electron decay mode search is presented in Sec. V. Conclusions are summarized in Sec. VI.

\section{THE APEX DETECTOR}

The APEX detector was located in a $15.9 \mathrm{~m}$ straight section of the Fermilab Antiproton Accumulator ring, which has a circumference of $474 \mathrm{~m}$. The detector was designed to identify $\bar{p}$ decays within a short $3.7 \mathrm{~m}$ long section of the ring, and was optimized to detect a single energetic charged track (electron or muon), originating from the beam, and accompanied by one or more neutral pions or photons. A schematic of the detector is shown in Fig. 1. In the following we give a brief description of the main detector components relevant to the analysis described in this paper. A more detailed description can be found in Ref. [10]. The APEX detector consisted of the following:

(i) A $3.7 \mathrm{~m}$ long evacuated decay tank operated at $10^{-11}$ Torr. The vacuum was maintained by a roughing pump, an ion diffusion pump, and 12 titanium sublimation filaments. The tank was designed to minimize the residual gas within the fiducial volume of the experiment, and hence minimize backgrounds from beam-gas interactions. The downstream section of the tank consists of a $96 \mathrm{~cm}$ diameter cylindrical shell supporting a $1.2 \mathrm{~mm}$ thick (0.073 radiation lengths) stainless steel window.

(ii) A movable tungsten wire target at the upstream end of the tank ( $z=0$ where, in the APEX coordinate system, $z$ is measured in the direction of the antiproton beam). The target could be inserted into the beam halo to produce a localized source of particles for aligning and calibrating the detector.

(iii) An upstream veto system consisting of horizontal and vertical scintillation counters arranged around the $10 \mathrm{~cm}$ diameter beam pipe immediately upstream of the tank and target. The counters covered a $1 \times 1 \mathrm{~m}^{2}$ area normal to the beam direction, and were used to reject tracks from upstream interactions.

(iv) Three planes of horizontal and three planes of vertical scintillation counters downstream of the tank. Each plane consisted of two $50 \times 100 \times 1.27 \mathrm{~cm}^{3}$ counters. The last planes of horizontal and vertical counters, the preradiator, were downstream of a 2.3 radiation length lead wall, providing an aid in identifying electrons and photons. The remaining counter planes were upstream of the lead, and provided pulse height information used to count the number of charged particles in an event $(d E / d x$ counters). The measured pulse height distribution arising from the passage of minimum ionizing particles (MIP's) through the $d E / d x$ counters is well described by a GEANT Monte Carlo simula- 


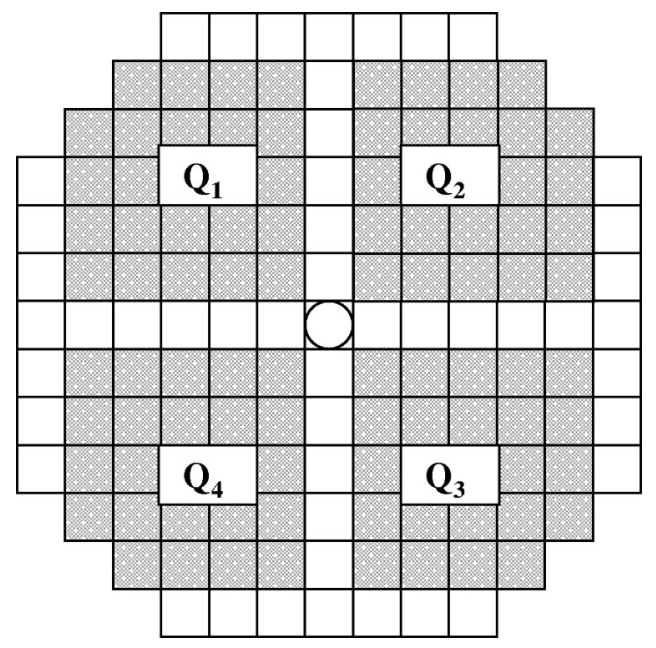

FIG. 2. The four calorimeter quadrants used for triggering.

tion in which the yield is 37 photoelectrons per MIP. The predicted efficiency for a minimum ionizing particle to produce a counter signal in excess of a 0.5 MIP threshold is $97 \%$.

(v) A lead-scintillator sampling electromagnetic calorimeter [11] constructed from 144 rectangular $10 \times 10 \mathrm{~cm}^{2}$ modules that are 14.7 radiation lengths deep. The modules were arranged in a $13 \times 13$ array with 6 modules removed from each of the four corners, and the central module removed to allow passage of the beam pipe (Fig. 2). Each module was read out with one $5.1 \mathrm{~cm}$ diameter photomultiplier. A light emitting diode (LED) pulser system enabled the stability of the photomultiplier gains for each module to be monitored. The calorimeter was calibrated by measuring the response to minimum ionizing tracks and reconstructing $\pi^{0} \rightarrow \gamma \gamma$ and $\eta \rightarrow \gamma \gamma$ mass peaks using data samples recorded with the calibration target inserted into the beam halo. The measured mass peaks have fractional rms widths given by $\sigma_{m} / \mathrm{m} \sim 0.25$ for cluster pairs in the energy range of interest for the experiment. This mass resolution is dominated by the energy resolution of the calorimeter.

(vi) A tail catcher (TC) downstream of the calorimeter consisting of a $20 \mathrm{~cm}$ deep lead wall followed by planes of horizontal and vertical scintillation counters. The TC aids in the identification of muon candidates where a signal consistent with a MIP is expected. In addition, the TC aids in the rejection of backgrounds to electron candidates such as caused by non-interacting hadrons.

(vii) A limited-acceptance muon telescope (MT), 10 nuclear interaction lengths deep, located downstream of the $\mathrm{TC}$, and aligned to point towards the center of the decay tank. The MT consists of a sandwich of five iron plates and five $30 \times 30 \mathrm{~cm}^{2}$ scintillation counters. The MT was used to identify charged particles that penetrate through several interaction lengths of material (muon candidates).

(viii) A tracking system consisting of three planes of horizontal and three planes of vertical $2 \mathrm{~mm}$ diameter scintillating fibers downstream of the tank and upstream of the preradiator lead. The fibers were measured to yield about 9 photoelectrons for a traversing minimum ionizing particle, and were read out using 12-stage Hamamatsu multianode photomultipliers. These detectors provided three space points along the track trajectory with typical residuals of $620 \mu \mathrm{m}$ in the directions transverse to the beam direction. This resolution enabled the origin of tracks emerging from the decay tank to be reconstructed with an rms precision given by $\sigma_{z}$ $=12 \mathrm{~cm}$. The average single-hit efficiency for a minimum ionizing particle passing through a tracking plane was $85 \%$. The measured track reconstruction efficiency, for a sample of events that are consistent with having one minimum ionizing particle passing through the $d E / d x$ planes and the MT, is $(90 \pm 7) \%$.

\section{DATA TAKING}

The APEX experiment took data during the April-July 1995 Fermilab Tevatron collider running period at times when there were typically $10^{12}$ antiprotons circulating in the Accumulator ring with a central $\bar{p}$ momentum of 8.90 $\pm 0.01 \mathrm{GeV} / c \quad(\gamma=9.54 \pm 0.01)$. APEX ran parasitically to the Fermilab Tevatron collider program. Because of increased background interactions when antiprotons were being stacked in the Accumulator, data were only taken during periods when stacking was not taking place. Such times occurred during the short period (typically $90 \mathrm{~min}$ ) before daily injection of antiprotons into the Tevatron, or at times when the collider complex was not running due to maintenance or repairs. A measure of the sensitivity of the APEX data sample is given by

$$
S \equiv \frac{1}{\gamma} \int N_{\bar{p}}^{-}(t) d t=(3.31 \pm 0.03) \times 10^{9} \mathrm{yr},
$$

where $N_{\bar{p}}(t)$ is the number of circulating antiprotons at time $t$, the integral is over the live-time of the experiment, and the uncertainty arises from the precision with which the time dependent beam current was recorded. Hence, if $\tau_{p}^{-}=3.31$ $\times 10^{9}$ years then on average 0.63 antiproton decays would have occurred somewhere within the Accumulator during the live-time of the experiment.

Energetic particles passing through the detector during Accumulator operation predominantly arise from interactions of the $\bar{p}$ beam with the residual gas in the decay tank or with material surrounding the beam. To suppress these backgrounds, and select candidate $\bar{p} \rightarrow \mu^{-} X$ and $\bar{p} \rightarrow e^{-} X$ decays, signals from the calorimeter and the scintillation counters were used to form muon and electron triggers.

\section{A. Muon trigger and data sample}

To search for muonic $\bar{p}$ decays, data were recorded with a muon trigger that required a coincidence between at least two of the five MT scintillation counters. These triggers were eliminated if they were in coincidence with a signal in one or more of the upstream veto counters indicating the presence of an interaction upstream of the decay tank. This loose trigger resulted in $1.2 \times 10^{6}$ events being recorded with a typical trigger rate of $\sim 1 \mathrm{~Hz}$. These events predominantly arise from background interactions in which the coincident MT counter signals are produced by traversing muons coming 
from charged pion decays, or by hadronic showers not contained in the calorimeter and TC.

\section{B. Electron trigger and data samples}

To search for electronic $\bar{p}$ decays, data were recorded with three complementary triggers: (i) An $E_{\text {balance }}$ trigger designed to select events consistent with a two-body $\bar{p}$ decay that results in the deposition of energy in two diagonally opposite quadrants of the calorimeter. The calorimeter cell groupings into four trigger quadrants are shown in Fig. 2. The trigger required the summed signals in each of two diagonally opposite quadrants to exceed a threshold that was set equal to about $2 \mathrm{GeV}$; (ii) An $E_{\text {total }}$ trigger designed to select $\bar{p}$ decays in which all of the decay products deposit approximately all of their energy within the calorimeter. This would result in a total calorimeter energy deposition $E_{\text {total }}$ close to the beam energy. The trigger therefore required the summed signals from all of the calorimeter cells to exceed a threshold that was set equal to about $7 \mathrm{GeV}$; and (iii) An electron trigger which required signals in the scintillators of the first two $d E / d x$ planes consistent with the passage of a MIP, a signal in the corresponding preradiator scintillators exceeding about $1.5 \mathrm{MIPs}$, and a signal in the corresponding calorimeter quadrant exceeding a summed energy threshold set equal to about $5 \mathrm{GeV}$.

All three triggers were combined with a signal from the upstream veto counters, used in anticoincidence to suppress events from upstream interactions. The typical data taking rates from background events satisfying the $E_{\text {balance }}, E_{\text {total }}$, and electron trigger requirements were respectively 3,9 , and $8 \mathrm{~Hz}$ when there were $10^{12}$ antiprotons circulating in the accumulator. The $E_{\text {balance }}, E_{\text {total }}$, and electron triggers resulted in a data sample of 37.8 million events recorded to tape for further analysis.

\section{Calibration triggers}

A number of additional triggers were used, either during normal data taking or during special running periods, to collect data for monitoring and calibrating the detector. Three of these triggers provided calibration data samples that were specially important for the analysis: (1) A very low threshold rate limited calorimeter trigger $\left(E_{\text {total }}>250 \mathrm{MeV}\right)$ that recorded events with minimal bias continuously during normal running. Dedicated minimal bias data samples were also taken during extended periods when there was a low intensity $\bar{p}$ current (corresponding to $\sim 10^{11}$ stored antiprotons) in the Accumulator; (2) A traversing charged track trigger that required a coincidence between the upstream veto counters and the TC counters. This trigger provided a sample of MIP's passing through the calorimeter; and (3) Low threshold $\left(E_{\text {total }}>5 \mathrm{GeV}\right)$ and high threshold $\left(E_{\text {total }}>7 \mathrm{GeV}\right)$ calorimeter triggers used during special periods during which the calibration target was inserted into the beam halo. This provided tracks coming from a known origin which facilitated the relative alignment of the tracking planes. These data also provided a sample of localized interactions which enabled the reconstruction of the $\pi^{0} \rightarrow \gamma \gamma$ and $\eta \rightarrow \gamma \gamma$ peaks used to define the calorimeter calibration.

\section{SEARCH FOR ANTIPROTON DECAY WITH A FINAL STATE MUON}

We begin with the sample of $1.2 \times 10^{6}$ events recorded with the muon trigger. Offline, after final calibration of the scintillation counters, the upstream veto counter requirement was re-applied using a more stringent threshold. This reduced the data sample to $1.1 \times 10^{6}$ events. The remaining backgrounds can be further suppressed by requiring that (a) the scintillation counter and scintillating fiber tracker signals are consistent with the presence of a minimum ionizing charged track that points back to the beam trajectory within the decay tank and that points forward to a pattern of minimum ionizing energy depositions within the MT counters, and (b) an event topology and kinematics that is consistent with the decay $\bar{p} \rightarrow \mu^{-}+X$. In the following, we consider first the simplest decay channel $\bar{p} \rightarrow \mu^{-} \gamma$, and then describe the search for more complicated decay modes.

\section{A. $\bar{p} \rightarrow \mu^{-} \gamma$ event selection}

To select events consistent with the decay $\bar{p} \rightarrow \mu^{-} \gamma$ we begin by requiring that (i) at least four of the five MT counters be above threshold $\left(4.2 \times 10^{4}\right.$ events), and (ii) there be one and only one reconstructed track that extrapolates to the MT counters within the expected uncertainty due to multiple scattering, and also extrapolates back to the beam orbit with a point of closest approach within the fiducial volume of the decay tank $(0<z<375 \mathrm{~cm})$ and with an impact parameter [10] less than $1 \mathrm{~cm}$ (416 events). Since a $\bar{p} \rightarrow \mu^{-} \gamma$ decay would result in events in which an energetic photon is produced coplanar with the muon (i.e. traveling within the plane defined by the muon and the incoming beam direction), we next require that (iii) there be at least one neutral cluster [10] in the calorimeter (209 events) that is coplanar with the muon candidate $\left( \pm 5^{\circ}\right)$. The data sample is reduced to 14 events. The observed neutral cluster energy distribution for these events is compared in Fig. 3 with the predicted distribution obtained using the GEANT [14] simulation described below, and corresponding to $\bar{p} \rightarrow \mu^{-} \gamma$ decay with a lifetime $\tau_{p}^{-} / B\left(\bar{p} \rightarrow \mu^{-} \gamma\right)=5000$ yr. The observed distribution peaks at low cluster energies, with a tail extending to approximately $2 \mathrm{GeV}$. In contrast, the predicted distribution for $\bar{p} \rightarrow \mu^{-} \gamma$ decays peaks at about $3.5 \mathrm{GeV}$, with only $4.4 \%$ of the simulated events having cluster energies less than 2 $\mathrm{GeV}$. We conclude that there is no evidence for a signal.

\section{B. Limit on $\bar{p} \rightarrow \mu^{-} \gamma$ decays}

To set a limit on $\tau_{p}^{-} / B\left(\bar{p} \rightarrow \mu^{-} \gamma\right)$ the remaining background events are eliminated by requiring that the neutral cluster energy exceed a minimum value $E_{\min }$, where we choose $E_{\min }=3 \mathrm{GeV}$. We note that although the choice of $E_{\min }$ is somewhat arbitrary, the final limit that we obtain on 


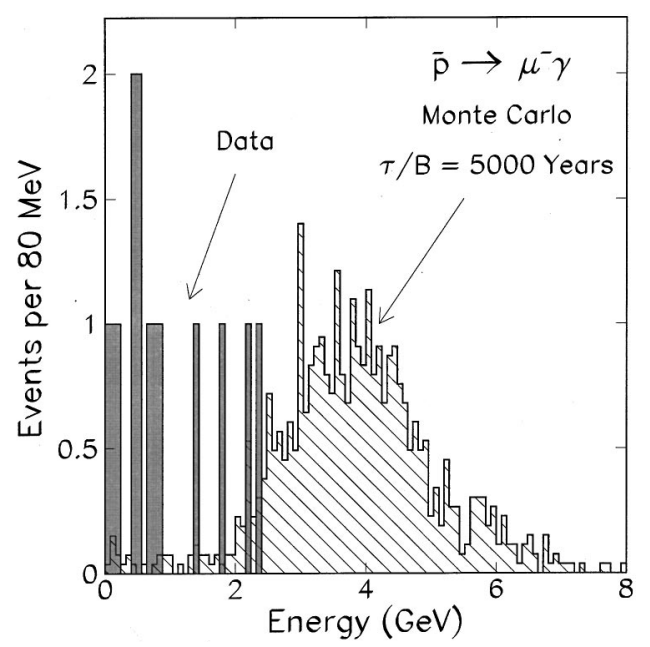

FIG. 3. Distribution of neutral cluster energies for the 14 events that pass the $\bar{p} \rightarrow \mu^{-} \gamma$ selection criteria described in the text (open histogram) compared with the predicted distribution for a signal corresponding to $\tau_{p}^{-} / B\left(\bar{p} \rightarrow \mu^{-} \gamma\right)=5000 \mathrm{yr}$ (hatched histogram).

the decay $\bar{p} \rightarrow \mu^{-} \gamma$ is insensitive to small changes in $E_{\text {min }}$. After setting $E_{\text {min }}=3 \mathrm{GeV}$, the resulting limit on $\tau_{p}^{-} / B(\bar{p}$ $\rightarrow \mu^{-} \gamma$ ) is given in years by

$$
\tau_{p}^{-} / B\left(\bar{p} \rightarrow \mu^{-} \gamma\right)>-\frac{1}{\ln \left(1-N_{\max } / \epsilon S\right)},
$$

where $N_{\max }=2.3$ is the $90 \%$ C.L. upper limit on the observation of $N=0$ events, and $\epsilon$ is the fraction of decays taking place uniformly around the accumulator ring that would pass the trigger and event selection requirements. If $\left(N_{\max } / \epsilon S\right)$ $\ll 1$, to a good approximation,

$$
\begin{aligned}
\tau_{p}^{-} / B\left(\bar{p} \rightarrow \mu^{-} \gamma\right) & >\frac{\epsilon}{\gamma} \frac{1}{N_{\max }} \int N_{\bar{p}}^{-}(t) d t \\
& =(3.31 \pm 0.03) \times 10^{9} \frac{\epsilon}{N_{\max }} .
\end{aligned}
$$

To take account of $\sigma_{\epsilon}$, the systematic uncertainty on $\epsilon$, we use the prescription given in Ref. [15], giving, at 90\% C.L.,

$$
N_{\max }=\mu_{\max } \times\left(1+\mu_{\max } \sigma_{r}^{2} / 2\right),
$$

where $\sigma_{r} \equiv \sigma_{\epsilon} / \epsilon$, and $\mu_{\max }$ is the upper limit corresponding to the observation of $N$ events $\left(\mu_{\max }=2.3\right.$ for $N=0$ ).

The GEANT Monte Carlo program has been used to simulate the detector response and calculate $\epsilon$. The detector simulation includes a full description of the detector geometry, and correctly describes the calorimeter, tracker, and scintillation counter responses $(d E / d x, \mathrm{MT})$ measured using calibration data, together with the measured performance of the muon trigger. Further details can be found in Ref. [10]. The efficiency $\epsilon$ was obtained by generating $10^{5} \bar{p} \rightarrow \mu^{-} \gamma$ decays uniformly along the beam orbit within the decay tank, and applying the trigger and data selection requirements to the simulated events. The geometrical efficiency of the de- tector and trigger is negligible for decays occurring outside of the tank. We obtain $\epsilon=(3.7 \pm 0.9) \times 10^{-5}$, where the uncertainty on $\epsilon$ arises from the systematic uncertainties on the muon trigger counter threshold calibration, the calorimeter energy scale, and the track reconstruction efficiency. The contributions to $\sigma_{r}$ arising from the uncertainties on the muon trigger counter threshold calibration and the calorimeter energy scale are, respectively, $\pm 18 \%$ and $\pm 15 \%$. These uncertainties have been evaluated by analyzing GEANT Monte Carlo $\bar{p} \rightarrow \mu^{-} \gamma$ samples in which the simulated trigger calibration and the calorimeter energy scale have been changed by \pm 1 standard deviation. The overall systematic uncertainty on $\epsilon$ was calculated by adding these contributions in quadrature with the uncertainty on the track reconstruction efficiency $( \pm 7 \%)$. Inserting the calculated $\epsilon$ and $\sigma_{\epsilon}$ into Eqs. (3) and (5) we obtain the result [13] $\tau_{p}^{-} / B(\bar{p}$ $\left.\rightarrow \mu^{-} \gamma\right)>5 \times 10^{4}$ yr (90\% C.L.).

Finally, we note that although our limit is insensitive to the exact value of $E_{\min }$, the dependence of the final result on the position of this last cut is somewhat unsatisfying. Hence, to verify our result, we have also used the more complicated statistical method described in Ref. [16]. In this alternative statistical analysis all of the events in the final $\left(E_{\text {min }}\right)$ distribution are retained, and use is made of how "signal-like" the position of each event is in the final distribution. Using this method we obtain a limit on $\tau_{p}^{-} / B\left(\bar{p} \rightarrow \mu^{-} \gamma\right)$ that is consistent with our quoted result.

\section{Search for $\bar{p} \rightarrow \mu^{-} \pi^{0}, \bar{p} \rightarrow \mu^{-} \eta$, and $\bar{p} \rightarrow \mu^{-} \gamma \gamma$ decays}

Now consider the two-body decays $\bar{p} \rightarrow \mu^{-} \pi^{0}$ and $\bar{p}$ $\rightarrow \mu^{-} \eta \quad\left(\pi^{0}, \quad \eta \rightarrow \gamma \gamma\right)$, and the three-body decay $\bar{p}$ $\rightarrow \mu^{-} \gamma \gamma$. These decays would result in events with one or two neutral clusters observed in the calorimeter, where the one-cluster events occur when the two photon showers are not spatially resolved in the calorimeter or when one of the photons is outside of the calorimeter acceptance. In addition, the $\eta$ may also decay into more complicated final states producing further clusters in the calorimeter. To optimize the search for $\mu^{-} \pi^{0}$ and $\mu^{-} \eta$ final states we divide the 209 events described previously that have a muon candidate plus one or more neutral clusters into two subsamples, namely a one-cluster sample containing 104 events, and a multi-cluster sample containing 105 events. The position of the cluster in the one-cluster sample is required to be coplanar with the muon $\left( \pm 10^{\circ}\right)$. The position of the cluster pair formed by the energy-weighted centroid of the two highest energy clusters in the multi-cluster events is also required to be coplanar with the muon $\left( \pm 10^{\circ}\right)$. These requirements reduce the samples to 13 one-cluster and 10 multi-cluster events. Note that we expect the multi-cluster events to contain 2 photons if they are genuine $\bar{p} \rightarrow \mu^{-} \pi^{0}, \mu^{-} \eta$, or $\mu^{-} \gamma \gamma$ decays. In most cases at least one of the two photons would be expected to convert in the preradiator lead. We therefore require that the preradiator counters in the quadrant opposite the MT have a signal exceeding a threshold of 0.5 MIP's. Only 3 


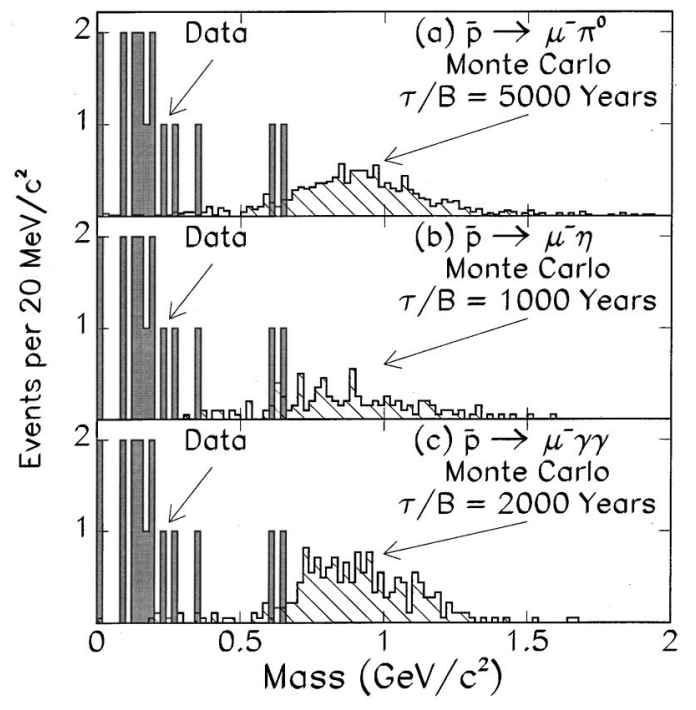

FIG. 4. Distribution of event masses for the 16 events that pass the selection criteria described in the text (open histograms) compared with predictions from a GEANT simulation (hatched histograms) for the decays (a) $\bar{p} \rightarrow \mu^{-} \pi^{0}$, (b) $\bar{p} \rightarrow \mu^{-} \eta$, and (c) $\bar{p}$ $\rightarrow \mu^{-} \gamma \gamma$. The two entries in the lowest bin are events with calorimeter energy less than the $\pi^{0}$ rest energy. The predicted signal distributions are normalized to correspond to $\tau_{p}^{-} / B=5000 \mathrm{yr}$ for the $\mu^{-} \pi^{0}$ mode, $1000 \mathrm{yr}$ for the $\mu^{-} \eta$ mode, and $2000 \mathrm{yr}$ for the $\mu^{-} \gamma \gamma$ mode.

multi-cluster events satisfy this requirement. Hence we are left with 16 events with $\geqslant 1$ cluster ( $\mu+\geqslant 1$ cluster events) for further analysis.

Under the hypothesis that the observed $(\mu \gamma)$ and $(\mu \gamma \gamma)$ systems arise from the decay of a beam particle, the mass of the parent particle can be computed from the measured muon direction and the directions and energies of the neutral clusters, using the constraint that the vector sum of the momentum components of the daughter particles transverse to the beam direction is zero. The resulting mass distribution for the remaining $16(\mu+\geqslant 1$ cluster) events is compared in Fig. 4 with predictions from the GEANT simulation for the decays (a) $\bar{p} \rightarrow \mu^{-} \pi^{0}$, (b) $\bar{p} \rightarrow \mu^{-} \eta$, and (c) $\bar{p} \rightarrow \mu^{-} \gamma \gamma$. The observed mass distribution peaks at low masses with a tail extending to approximately $0.7 \mathrm{GeV} / \mathrm{c}^{2}$. In contrast to this, the simulated signal distributions peak at the $\bar{p}$ mass, with only $17 \%(23 \%)[13 \%]$ of the simulated $\mu^{-} \pi^{0}\left(\mu^{-} \eta\right)$ $\left[\mu^{-} \gamma \gamma\right]$ decays resulting in reconstructed masses less than $0.7 \mathrm{GeV} / c^{2}$. We conclude that there is no evidence for a signal.

To set limits on $\tau_{p}^{-} / B$ we eliminate the remaining background by requiring that the reconstructed mass exceed a minimum value $m_{\min }$, and choose $m_{\min }=0.75 \mathrm{GeV} / c^{2}$. We note that although the choice of $m_{\min }$ is somewhat arbitrary, the final limits that we obtain on the decays $\bar{p} \rightarrow \mu^{-} \pi^{0}$, $\mu^{-} \eta$, and $\mu^{-} \gamma \gamma$ are insensitive to small changes in $m_{\min }$. The calculated overall efficiencies $\epsilon$ for these decays to pass our trigger and analysis requirements are $(3.6 \pm 0.9) \times 10^{-5}$ for the $\mu^{-} \pi^{0}$ mode, $(6.1 \pm 1.9) \times 10^{-6}$ for the $\mu^{-} \eta$ mode, and $(1.8 \pm 0.5) \times 10^{-5}$ for the $\mu^{-} \gamma \gamma$ mode. Substituting the values into Eqs. (3) and (5) yields the limits $\tau_{p}^{-} / B(\bar{p}$ $\left.\rightarrow \mu^{-} \pi^{0}\right)>5 \times 10^{4} \mathrm{yr}, \quad \tau_{p}^{-} / B\left(\bar{p} \rightarrow \mu^{-} \eta\right)>8 \times 10^{3} \mathrm{yr}$, and $\tau_{p}^{-} / B\left(\bar{p} \rightarrow \mu^{-} \gamma \gamma\right)>2 \times 10^{4}$ yr at $90 \%$ C.L.

Finally, to verify our result, we have removed the last cut on our final (mass) distribution, and used the more complicated statistical method described in Ref. [16]. The resulting limits on the $\mu^{-} \pi^{0}, \mu^{-} \eta$, and $\mu^{-} \gamma \gamma$ decay modes are consistent with our quoted results.

\section{Other $\bar{p} \rightarrow \mu^{-}+X$ decays}

Now consider the other possible two-body muonic decay modes of the antiproton, namely decays into the final states $\mu^{-} K_{S}^{0}, \mu^{-} K_{L}^{0}, \mu^{-} \rho^{0}$, and $\mu^{-} \omega$. Detailed GEANT simulations have been made for these decay modes. The calculated geometrical acceptances for the $\mu^{-} \rho^{0}$ and $\mu^{-} \omega$ modes are very small. We therefore restrict ourselves to the $\mu^{-} K_{S}^{0}$ and $\mu^{-} K_{L}^{0}$ modes. We have not made an explicit event selection for the $\mu^{-} K_{S}^{0}$ and $\mu^{-} K_{L}^{0}$ modes. However, the GEANT simulations predict the fraction of these decays that would have passed our $\bar{p} \rightarrow \mu^{-} \gamma$ and $\bar{p} \rightarrow \mu^{-} \pi^{0}$ selection requirements. We can use these calculated efficiencies to set an upper limit on the $\mu^{-} K_{S}^{0}$ and $\mu^{-} K_{L}^{0}$ modes. The calculated efficiencies $\epsilon$ for these decays to satisfy the trigger and either the $\mu^{-} \gamma$ or $\mu^{-} \pi^{0}$ search criteria described previously are (3.3 \pm 1.0$)$ $\times 10^{-6}$ for the $\mu^{-} K_{S}^{0}$ mode, and $(5.0 \pm 1.5) \times 10^{-6}$ for the $\mu^{-} K_{L}^{0}$ mode. Substituting these values into Eqs. (3) and (5) yields the limits $\tau_{p}^{-} / B\left(\bar{p} \rightarrow \mu^{-} K_{S}^{0}\right)>4 \times 10^{3}$ yr and $\tau_{p}^{-} / B(\bar{p}$ $\left.\rightarrow \mu^{-} K_{L}^{0}\right)>7 \times 10^{3}$ yr at $90 \%$ C.L. To verify these results we have removed the last cut on our final kinematic distribution and used the more complicated statistical method described in Ref. [16]. The resulting limits on the $\mu^{-} K_{S}^{0}$ and $\mu^{-} K_{L}^{0}$ decay modes are consistent with our quoted results.

\section{SEARCH FOR ANTIPROTON DECAY WITH A FINAL STATE ELECTRON}

We begin with the sample of $37.8 \times 10^{6}$ events recorded with the $E_{\text {balance }}, E_{\text {total }}$, and electron triggers described in Sec. III B. Offline, after final calibration of the scintillation counters, a re-application of the upstream veto counter requirement reduces this sample to $34.2 \times 10^{6}$ events. The remaining backgrounds can be suppressed by requiring that the signals from the tracker, calorimeter, and scintillation counters are consistent with events arising from $\bar{p} \rightarrow e^{-}+X$ decays. In the following we first consider the simplest decay channel $\bar{p} \rightarrow e^{-} \gamma$, and then describe the search for more complicated decay modes.

\section{A. $\bar{p} \rightarrow e^{-} \gamma$ event selection}

To select events consistent with the decay $\bar{p} \rightarrow e^{-} \gamma$ we begin by requiring the following offline selection:

(1) A pattern of hits in the $d E / d x$ counters consistent with the presence of one, and only one, charged track (16.9 $\times 10^{6}$ events). 
(2) A pulse height in excess of 1.5 MIP's in the preradiator counters $\left(9.6 \times 10^{6}\right.$ events $)$. This is a first, very loose, electron candidate requirement.

(3) No signal in excess of 0.6 MIP's in both a horizontal and vertical TC counter $\left(7.6 \times 10^{6}\right.$ events). Note that electrons and photons are expected to produce electromagnetic showers that are fully contained within the calorimeter and the TC lead wall, and do not penetrate into the TC.

(4) Either 2 or 3 clusters reconstructed in the calorimeter $\left(2.5 \times 10^{6}\right.$ events $)$. A fully contained $\bar{p} \rightarrow e^{-} \gamma$ event would normally result in two calorimeter clusters. However a third cluster can arise if, for example, a large angle photon is radiated from the electron as it passes through the preradiator.

(5) More than $90 \%$ of the total energy recorded in the calorimeter be outside of the cells immediately surrounding the beampipe $\left(1.3 \times 10^{6}\right.$ events). This requirement suppresses backgrounds associated with beam halo outside of, but close to, the beampipe. The requirement also ensures that a significant fraction of the energy associated with the observed electromagnetic showers has not "leaked" out of the calorimeter into the uninstrumented region within the beampipe.

These very loose selection criteria were designed to reduce the sample to a reasonable size before running the track reconstruction code. After track reconstruction we impose the following requirements:

(6) There must be one and only one reconstructed track that extrapolates to a calorimeter cluster $\left(1.0 \times 10^{6}\right.$ events).

(7) The reconstructed track must be consistent with originating from within the fiducial volume of the decay tank. Specifically, the point of closest approach of the track to the beamline must be in the region $0<z<375 \mathrm{~cm}\left(2.7 \times 10^{5}\right.$ events).

(8) The distance of closest approach of the reconstructed track to the beamline [10] must be less than $1 \mathrm{~cm}(6.0$ $\times 10^{4}$ events).

(9) There must be a preradiator signal that exceeds 4 MIP's $\left(2.2 \times 10^{4}\right.$ events). This requirement was based on a study of the preradiator signals associated with the $\pi^{0}$ $\rightarrow \gamma \gamma$ calibration data samples.

(10) The electron candidate must be coplanar $(\Delta \phi$ $>3.05$ radians) with the beamline and the highest energy neutral cluster (467 events).

Figures 5(a) and 5(b) compare, respectively, the transverse-momentum-balance and total-energy distributions for these 467 events with predictions from a GEANT simulation of $\bar{p} \rightarrow e^{-} \gamma$ decays. The observed distributions have peaks corresponding to large transverse-momentum imbalances and low total energies. In contrast to this, the simulated signal distributions peak at small transverse-momentum imbalance and at total energies corresponding to the beam energy. The backgrounds can therefore be further suppressed by requiring that candidate events are kinematically consistent with $\bar{p} \rightarrow e^{-} \gamma$ decays.

\section{B. $\bar{p} \rightarrow e^{-} \gamma$ kinematics and a limit on $\tau / B\left(\bar{p} \rightarrow e^{-} \gamma\right)$}

In general, a two-body decay in which the daughter particles have known masses can be completely specified by 9
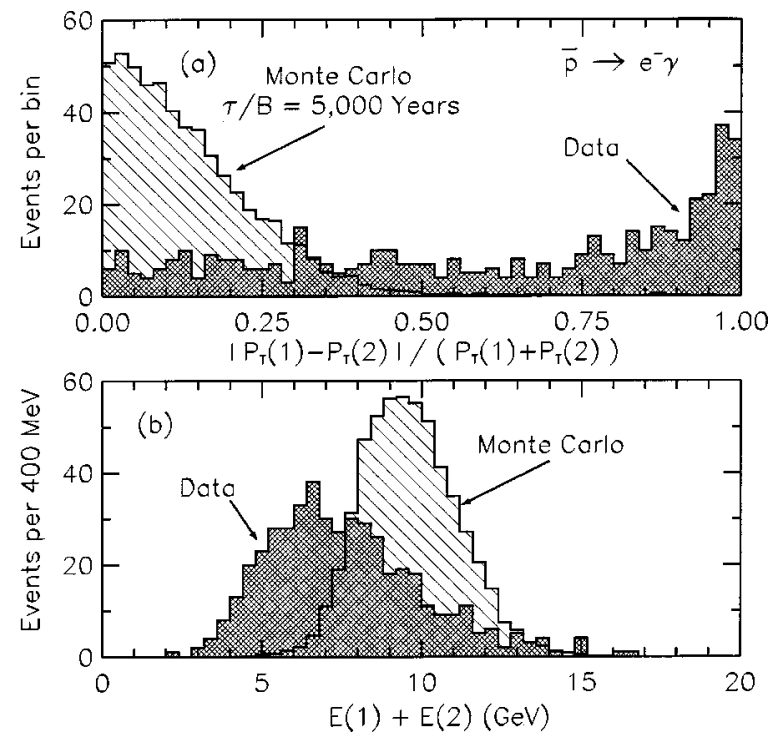

FIG. 5. Kinematic distributions for the $467 \bar{p} \rightarrow e^{-} \gamma$ candidate events prior to the kinematic analysis described in the text. The observed distributions are compared with GEANT Monte Carlo predictions for $\bar{p} \rightarrow e^{-} \gamma$ decays normalized to correspond to a lifetime of 5000 years, and shown for (a) a measure of the transverse momentum balance in the event, where $P_{T}(1)$ and $P_{T}(2)$ are the transverse momenta of the two highest energy clusters in the event, and (b) the sum of the energies of the two highest energy clusters in the event.

parameters (two momentum vectors and a decay point $\left.x_{0}, y_{0}, z_{0}\right)$. Consider $\bar{p} \rightarrow e^{-} \gamma$ decays in which the parent antiprotons are traveling along the beamline with momenta $p_{0}$, energy $E_{0}$. In this case there are 6 constraints: (i) $x_{0}=0$, (ii) $y_{0}=0$, (iii) $E(e)+E(\gamma)=E_{0}$, (iv) $p_{x}(e)+p_{x}(\gamma)=0$, (v) $p_{y}(e)+p_{y}(\gamma)=0$, and (vi) $p_{z}(e)+p_{z}(\gamma)=p_{0}$. Hence, to completely specify a $\bar{p} \rightarrow e^{-} \gamma$ decay occurring within the APEX decay tank, we need to specify the values of 3 parameters, which we choose to be $z_{0}, E(e)$, and the $x$ coordinate of the intersection of the electron trajectory with the calorimeter $x_{c a l}(e)$. In the following we will refer to a specific set of values for these parameters as a decay hypothesis. For a given decay hypothesis the intersections of the electron track with the 3 horizontal and 3 vertical tracking planes can be predicted, along with the positions and energies of the calorimeter clusters associated with the electron and photon. To quantify how well a given decay hypothesis describes the observed locations of the hits in the tracker planes, and the observed locations and energies of the clusters in the calorimeter, we define

$$
\chi^{2} \equiv \sum_{i=1}^{n}\left[\frac{\Delta_{i}}{\sigma_{i}}\right]^{2}
$$

where the sum is over the 6 calorimeter measurements ( $[x, y]$ positions and energies for the electron and photon clusters) and $(n-6)$ tracking measurements. The $\Delta_{i}$ are the differences between the measured and predicted values for the $i$ th measurement, and the $\sigma_{i}$ are the corresponding mea- 


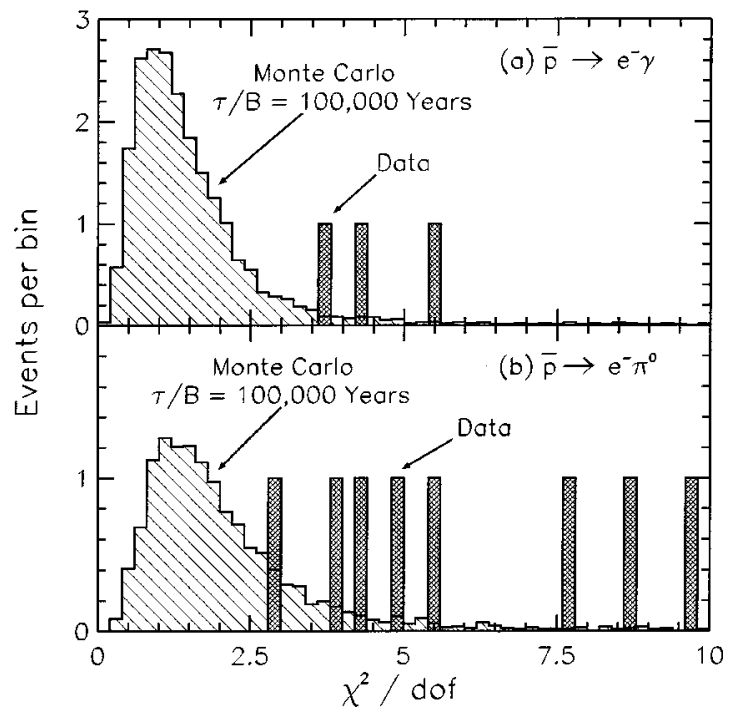

FIG. 6. Distribution of $\chi^{2}$ per degree of freedom for (a) the 3 $\bar{p} \rightarrow e^{-} \gamma$ candidate events surviving the kinematic fitting described in the text, compared with the GEANT Monte Carlo predictions for $\bar{p} \rightarrow e^{-} \gamma$ decays normalized to correspond to a lifetime of 100000 years, and (b) the $8 \bar{p} \rightarrow e^{-} \pi^{0}$ candidate events surviving the kinematic fitting described in the text, compared with the GEANT Monte Carlo predictions for $\bar{p} \rightarrow e^{-} \pi^{0}$ decays normalized to correspond to a lifetime of 100000 years.

surement uncertainties. Note that the track reconstruction code requires that at least 4 of the 6 scintillating fiber planes record a hit along the track, and hence the number of measurements used in the $\chi^{2}$ sum is in the range $10 \leqslant n \leqslant 12$. The best decay hypothesis for a given event is found by finding the set of parameters $\left[z_{0}, E(e)\right.$, and $\left.x_{c a l}(e)\right]$ that minimizes the $\chi^{2}$ per degree of freedom $\left(\chi^{2} / \mathrm{dof}=\chi^{2} / n\right)$. In this fitting procedure, hypotheses are rejected if the $\chi^{2} /$ dof $>10$. Only 3 of the 467 events shown in Fig. 5(a) have a best-fit decay hypothesis that survives this loose kinematic requirement. In Fig. 6(a) the distribution of the fitted values of $\chi^{2} /$ dof for these events are compared with the predicted GEANT Monte Carlo distribution for $\bar{p} \rightarrow e^{-} \gamma$ decays that pass the trigger and offline selection requirements. There is no evidence for a signal. The observed events have values of $\chi^{2} /$ dof larger than would be expected for typical $\bar{p} \rightarrow e^{-} \gamma$ decays.

To set a limit on $\tau / B\left(\bar{p} \rightarrow e^{-} \gamma\right)$ we require that the $\chi^{2} /$ dof for the best-fit hypothesis is less than a maximum value, which we set equal to 2 . No events survive this requirement. The efficiency for $\bar{p} \rightarrow e^{-} \gamma$ decays to pass the trigger and offline selection requirements has been evaluated using the GEANT Monte Carlo program to generate $10^{5}$ simulated decays uniformly along the beamline within the decay tank. We find $\epsilon=(5.2 \pm 1.4) \times 10^{-4}$, where the uncertainty on $\epsilon$ arises from the systematic uncertainties on the trigger calibration, calorimeter energy scale, track reconstruction efficiency, and Monte Carlo statistics. The trigger and calorimeter scale uncertainties yield contributions to $\sigma_{r}$ of $\pm 14 \%$ and $\pm 21 \%$, respectively, and have been evaluated by analyzing GEANT Monte Carlo $\bar{p} \rightarrow e^{-} \gamma$ samples in which the simulated trigger and calorimeter scales have been changed by $\pm 1 \sigma$. The overall systematic uncertainty on $\epsilon$ was calculated by adding these contributions in quadrature with the uncertainty on the track reconstruction efficiency $( \pm 7 \%)$ and Monte Carlo statistics ( $\pm 1 \%)$. Inserting the calculated $\epsilon$ and $\sigma_{\epsilon}$ into Eqs. (3) and (5) we obtain the result $\tau_{p}^{-} / B(\bar{p}$ $\left.\rightarrow e^{-} \gamma\right)>7 \times 10^{5}$ yr $(90 \%$ C.L.). To verify this result we have removed the cut on the $\chi^{2} /$ dof distribution and used the more complicated statistical method described in Ref. [16]. The resulting limit on $\tau_{p}^{-} / B\left(\bar{p} \rightarrow e^{-} \gamma\right)$ is consistent with our quoted result.

\section{Search for $\bar{p} \rightarrow e^{-} \pi^{0}$}

To select events consistent with the decay $\bar{p} \rightarrow e^{-} \pi^{0}$ we begin by applying the $\bar{p} \rightarrow e^{-} \gamma$ selection requirements (1)(9) described in Sec. V A. These requirements select 2.2 $\times 10^{4}$ events in which there is a candidate electron together with 1 or 2 additional clusters in the calorimeter. We would expect genuine $\bar{p} \rightarrow e^{-} \pi^{0}$ decays to be either reconstructed as an electron plus one additional cluster (if the two photons from the $\pi^{0}$ decay are not spatially resolved in the calorimeter), or as an electron plus two additional clusters (if the two photons are resolved). If there are two additional clusters, we require the cluster-cluster mass to be less than $500 \mathrm{MeV} / \mathrm{c}^{2}$ $\left(2.1 \times 10^{4}\right.$ events). It is convenient to analyze all the $\bar{p}$ $\rightarrow e^{-} \pi^{0}$ candidate events as two-body decays. To do this we reduce the electron plus two cluster events to an electron plus one cluster topology by combining the two neutral clusters. The combined cluster has an energy equal to the sum of the energies of the component clusters, and is located in the $(x, y)$ plane at a position given by the energy-weighted centroid of the component clusters. We now require that the electron candidate is coplanar $(\Delta \phi>2.9$ radians) with the beamline and the neutral cluster (1091 events). Applying the two-body kinematics fitting procedure described in Sec. V B, only 8 events have $\chi^{2} /$ dof $<10$. In Fig. 6 the distribution of the fitted values of $\chi^{2} /$ dof for these events are compared with the predicted GEANT Monte Carlo distribution for $\bar{p}$ $\rightarrow e^{-} \pi^{0}$ decays that pass the trigger and offline selection requirements. There is no evidence for a signal. The observed events have values of $\chi^{2} /$ dof larger than would be expected for $\bar{p} \rightarrow e^{-} \pi^{0}$ decays.

To set a limit on $\tau / B\left(\bar{p} \rightarrow e^{-} \pi^{0}\right)$ we require that the $\chi^{2} /$ dof for the best-fit hypothesis is less than a maximum value, which we set equal to 2.5 . No events survive this requirement. The efficiency for $\bar{p} \rightarrow e^{-} \pi^{0}$ decays to pass the trigger and offline selection requirements has been evaluated using the GEANT Monte Carlo program to generate $10^{5}$ simulated decays uniformly along the beamline within the decay tank. We find $\epsilon=(3.0 \pm 0.7) \times 10^{-4}$, where the uncertainty on $\epsilon$ arises from the systematic uncertainties on the trigger calibration, calorimeter energy scale, track reconstruction efficiency, and Monte Carlo statistics. Inserting the calculated $\epsilon$ and $\sigma_{\epsilon}$ into Eqs. (3) and (5) we obtain the result $\tau_{p}^{-} / B(\bar{p}$ $\left.\rightarrow e^{-} \pi^{0}\right)>4 \times 10^{5}$ yr $(90 \%$ C.L.). To verify this result, we have removed the cut on the $\chi^{2} /$ dof distribution, and used 
the more complicated statistical method described in Ref. [16]. The resulting limit on $\tau_{p}^{-} / B\left(\bar{p} \rightarrow e^{-} \pi^{0}\right)$ is consistent with our quoted result. We have also searched for $\bar{p}$ $\rightarrow e^{-} \pi^{0}$ decays by employing an analysis in which the twobody fitting procedure is replaced by a series of cuts on kinematic distributions [17]. This alternative analysis also yields a limit on $\tau_{p}^{-} / B\left(\bar{p} \rightarrow e^{-} \pi^{0}\right)$ similar to our quoted result.

\section{Search for $\bar{p} \rightarrow e^{-} \eta, e^{-} K_{S}^{0}, e^{-} \omega$, and $e^{-} \gamma \gamma$}

We begin by describing the search for $\bar{p} \rightarrow e^{-} \eta$. The selection requirements employed for this decay mode are also used for the search for the $e^{-} K_{S}^{0}, e^{-} \omega$, and $e^{-} \gamma \gamma$ final states. The results for these latter decay modes are presented in Sec. V D 2.

$$
\text { 1. } \bar{p} \rightarrow e^{-} \eta
$$

The $\eta$ decays into neutral modes $\left(\eta \rightarrow 2 \gamma, 3 \pi^{0}, \pi^{0} 2 \gamma\right)$ with a branching fraction of $\sim 0.7$. The decay $\bar{p} \rightarrow e^{-} \eta$, with the $\eta$ subsequently decaying into these neutral modes, would give rise to an electron plus two-or-more photons in the final state. To select events consistent with the decay $\bar{p} \rightarrow e^{-} \eta$ we begin with the $\bar{p} \rightarrow e^{-} \gamma$ selection requirements (1)-(9) described in Sec. V A, which yield the sample of $2.2 \times 10^{4}$ events in which there is a candidate electron together with 1 or 2 additional clusters in the calorimeter. We then require that

(i) There are two clusters in addition to the electron candidate $\left(1.2 \times 10^{4}\right.$ events).

(ii) The lowest energy neutral cluster has an energy exceeding $1 \mathrm{GeV}$ (1222 events), and the other two clusters both have energies in the range $2<E<6 \mathrm{GeV}$ (394 events).

(iii) The electron candidate is coplanar ( $\Delta \phi>2.8$ radians) with the beamline and the neutral cluster pair (46 events). The coplanarity requirement is based upon a GEANT Monte Carlo study of simulated $\bar{p} \rightarrow e^{-} \eta$ decays within the APEX decay tank, and is less stringent than the corresponding requirements used for the $\bar{p} \rightarrow e^{-} \gamma$ and $\bar{p} \rightarrow e^{-} \pi^{0}$ searches described previously.

(iv) The total energy measured in the calorimeter is in the range $7<E_{\text {tot }}<13 \mathrm{GeV}$ (30 events).

(v) The transverse-momentum-balance variable $p_{T}^{\text {bal }}$ is less than 0.3 , where $p_{T}^{b a l} \equiv\left|p_{t}(e)-p_{t}(\gamma \gamma)\right| /\left[p_{t}(e)\right.$ $\left.+p_{t}(\gamma \gamma)\right](24$ events).

(vi) The event lies within the dashed region of the $\left(M_{X}^{2}\right.$, $M_{12}$ ) plane shown in Fig. 7 ( 2 events), where the missingmass-squared $\left(M_{X}^{2}\right)$ is computed using the incoming $\bar{p}$ four vector and the outgoing electron candidate four vector, and $M_{12}$ is the measured mass of the cluster pair. The region accepted contains $86 \%$ of the simulated $\bar{p} \rightarrow e^{-} \eta$ decays that survive the previous selection requirements.

The distribution of the observed events in the $\left(M_{X}^{2}, M_{12}\right)$ plane is shown in Fig. 7(a) for the 24 events that survive the selection criteria up to (but not including) the $\left(M_{X}^{2}, M_{12}\right)$ requirement. The event population suggests that the 2 events

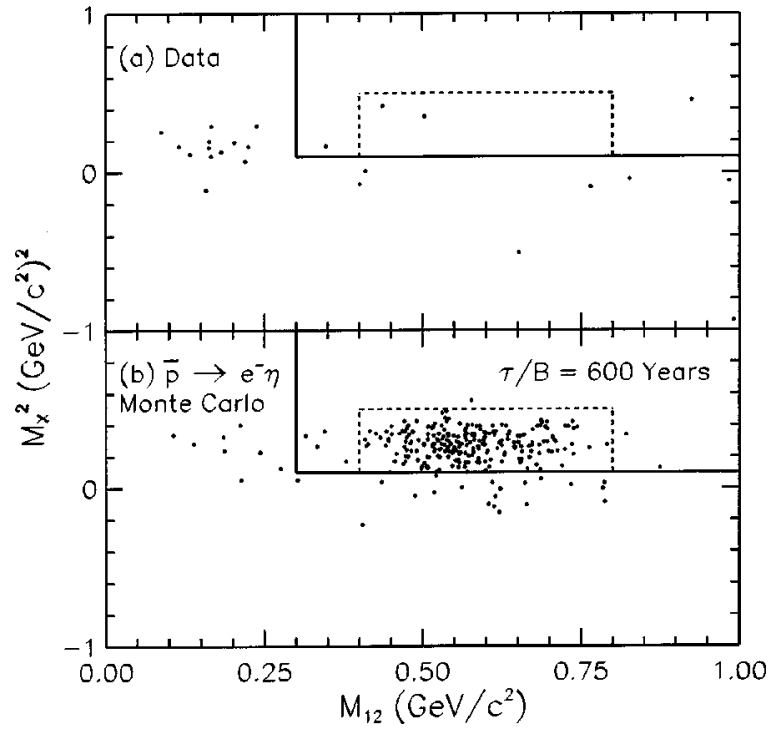

FIG. 7. Observed event populations in the (a) missing-masssquared versus cluster-cluster mass plane for candidate events, and (b) simulated $\bar{p} \rightarrow e^{-} \eta$ decays that pass the prior selection requirements described in the text. The simulated data correspond to a lifetime of 600 years. The dashed lines delineate the region accepted by the analysis cuts for $\bar{p} \rightarrow e^{-} \eta$ decays and the solid lines delineate the region accepted by the analysis cuts for other modes, as described in the text.

that survive the $\left(M_{X}^{2}, M_{12}\right)$ requirement are associated with the high-mass tail of the background distribution. However, we cannot distinguish these events from genuine $\bar{p} \rightarrow e^{-} \eta$ decays, and hence our limit on this decay mode is based on the observation of 2 events, yielding $\mu_{\max }=5.7$ at $90 \%$ C.L. The efficiency for $\bar{p} \rightarrow e^{-} \eta$ decays to pass the trigger and offline selection requirements has been evaluated using the GEANT Monte Carlo program to generate $6 \times 10^{4}$ simulated decays uniformly along the beamline within the decay tank. We find $\epsilon=(4.4 \pm 1.1) \times 10^{-5}$, where the uncertainty on $\epsilon$ arises from the systematic uncertainties on the trigger calibration, calorimeter energy scale, track reconstruction efficiency, and Monte Carlo statistics. Inserting the calculated $\epsilon$ and $\sigma_{\epsilon}$ into Eqs. (3) and (5) we obtain the result $\tau_{p}^{-} / B(\bar{p}$ $\left.\rightarrow e^{-} \eta\right)>2 \times 10^{4}$ yr $(90 \%$ C.L. $)$.

$$
\text { 2. } \bar{p} \rightarrow e^{-} K_{S}^{0}, e^{-} \omega \text {, and } e^{-} \gamma \gamma
$$

The selection requirements defined for the $\bar{p} \rightarrow e^{-} \eta$ search will also select candidates for other two-body $\bar{p}$ decay modes with an electron in the final state, provided the neutral particle accompanying the electron (i) is sufficiently massive to produce events that satisfy an $\left(M_{X}^{2}, M_{12}\right)$ requirement, and (ii) decays at least some of the time into daughter particles that are observed as two neutral clusters. In particular, the $\bar{p} \rightarrow e^{-} \eta$ search results enable us to place limits on the decay modes $\bar{p} \rightarrow e^{-} K_{S}^{0}, e^{-} \omega$, and $e^{-} \gamma \gamma$. For these modes we require candidate events that fall in the region defined by $0.1<M_{X}^{2}<1.0 \mathrm{GeV}^{2} / c^{4}$ and $0.3<M_{12}<1.0 \mathrm{GeV} / c^{2}$ (Fig. $7)$. The corresponding limits will be based upon the obser- 


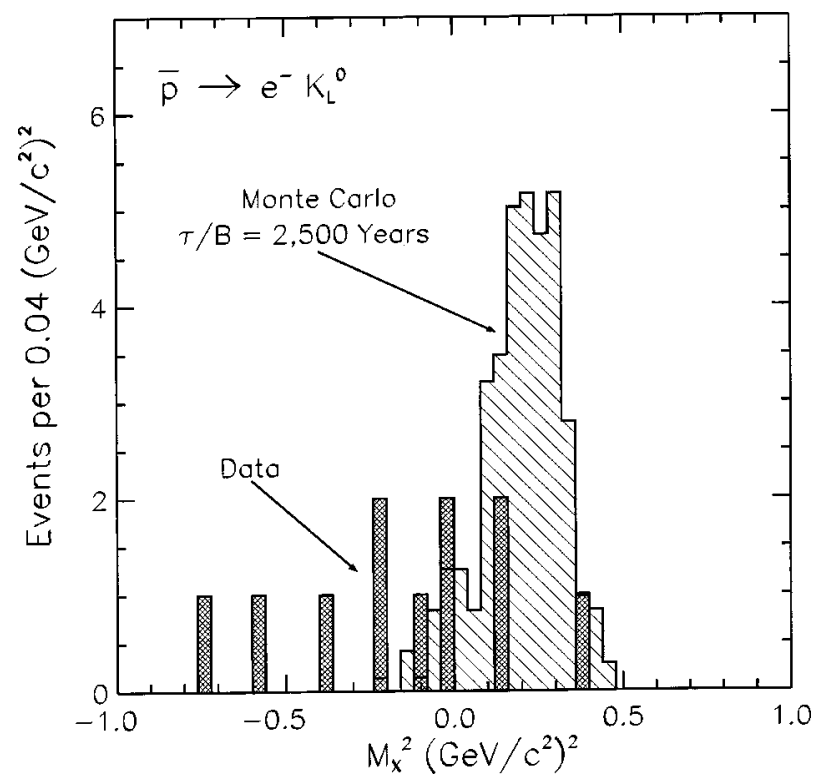

FIG. 8. Distribution of missing-mass-squared for the $11 \bar{p}$ $\rightarrow e^{-} K_{L}^{0}$ candidate events that survive all of the requirements described in the text except for the missing-mass-squared requirement. The observed distributions are compared with the GEANT Monte Carlo predictions for $\bar{p} \rightarrow e^{-} K_{L}^{0}$ decays normalized to correspond to a lifetime of 2500 years.

vation of 4 events, yielding $\mu_{\max }=8.9$ at $90 \%$ C.L. Note that, of the simulated signal events that pass all the prior selection requirements, the final $\left(M_{X}^{2}, M_{12}\right)$ requirement accepts $45 \%$ of the simulated $e^{-} K_{S}^{0}$ decays, $70 \%$ of the $e^{-} \omega$ decays, and $57 \%$ of the $\bar{p} \rightarrow e^{-} \gamma \gamma$ decays. The efficiencies for these decays to pass the trigger and offline selection requirements have been evaluated using the GEANT Monte Carlo program, and are given by $\epsilon=(3.0 \pm 0.9) \times 10^{-6}$ for the $e^{-} K_{S}^{0}$ mode, $\epsilon=(6.8 \pm 1.9) \times 10^{-7}$ for the $e^{-} \omega$ mode, and $\epsilon=(4.9 \pm 1.3) \times 10^{-5}$ for the $e^{-} \gamma \gamma$ mode. Inserting the calculated values for $\epsilon$ and $\sigma_{\epsilon}$ into Eqs. (3) and (5), at $90 \%$ C.L. we obtain the results $\tau_{p}^{-} / B\left(\bar{p} \rightarrow e^{-} X\right)>900 \mathrm{yr}$ for the $e^{-} K_{S}^{0}$ mode, $200 \mathrm{yr}$ for the $e^{-} \omega$ mode, and $2 \times 10^{4} \mathrm{yr}$ for the $e^{-} \gamma \gamma$ mode.

\section{E. Search for $\bar{p} \rightarrow e^{-} K_{L}^{0}$}

A $K_{L}^{0}$ with an energy of a few $\mathrm{GeV}$ will travel of order $100 \mathrm{~m}$ before decaying. Hence, $\bar{p} \rightarrow e^{-} K_{L}^{0}$ decays occurring within the APEX fiducial volume would be expected to produce an electron accompanied by one neutral cluster in the calorimeter. In our search for $\bar{p} \rightarrow e^{-} K_{L}^{0}$ decays we begin with the $\bar{p} \rightarrow e^{-} \gamma$ selection requirements (1)-(9) described in Sec. V A, which yield the sample of $2.2 \times 10^{4}$ events in which there is a candidate electron together with 1 or 2 additional clusters in the calorimeter. We then require that

(i) There is only one cluster in addition to the electron candidate (9300 events).

(ii) The electron candidate is coplanar $(\Delta \phi>3$ radians) with the beamline and the neutral cluster (300 events).
TABLE I. Summary of results: $90 \%$ C.L. limits on $\tau / B$ for 13 antiproton decay modes.

\begin{tabular}{cccc}
\hline \hline Decay mode & $\begin{array}{c}\tau / B \text { limit } \\
\text { (years) }\end{array}$ & Decay mode & $\begin{array}{c}\tau / B \text { limit } \\
\text { (years) }\end{array}$ \\
\hline$\mu^{-} \gamma$ & $5 \times 10^{4}$ & $e^{-} \gamma$ & $7 \times 10^{5}$ \\
$\mu^{-} \pi^{0}$ & $5 \times 10^{4}$ & $e^{-} \pi^{0}$ & $4 \times 10^{5}$ \\
$\mu^{-} \eta$ & $8 \times 10^{3}$ & $e^{-} \eta$ & $2 \times 10^{4}$ \\
$\mu^{-} \gamma \gamma$ & $2 \times 10^{4}$ & $e^{-} \gamma \gamma$ & $2 \times 10^{4}$ \\
$\mu^{-} K_{L}^{0}$ & $7 \times 10^{3}$ & $e^{-} K_{L}^{0}$ & $9 \times 10^{3}$ \\
$\mu^{-} K_{S}^{0}$ & $4 \times 10^{3}$ & $e^{-} K_{S}^{0}$ & $9 \times 10^{2}$ \\
& & $e^{-} \omega$ & $2 \times 10^{2}$ \\
\hline \hline
\end{tabular}

(iii) The total energy measured in the calorimeter is in the range $7<E_{\text {tot }}<13 \mathrm{GeV}$ (157 events).

(iv) The electron cluster and the neutral cluster both have energies in the range $2<E<6 \mathrm{GeV}$ (11 events).

(v) The missing-mass-squared lies in the range $0.1<M_{X}^{2}$ $<0.4 \mathrm{GeV}^{2} / c^{4}$, where $M_{X}^{2}$ is computed using the incoming $\bar{p}$ four vector and the outgoing electron candidate four vector (3 events).

The $M_{X}^{2}$ distribution for the observed events is shown in Fig. 8 for the 11 events that survive the selection criteria up to (but not including) the $M_{X}^{2}$ requirement. The event population suggests that the 3 events that survive the $M_{X}^{2}$ requirement are associated with the high-mass tail of the background distribution. However, we cannot distinguish these events from genuine $\bar{p} \rightarrow e^{-} K_{L}^{0}$ decays, and hence our limit on this decay mode is based on the observation of 3 events, yielding $\mu_{\max }=7.3$ at $90 \%$ C.L. The efficiency for $\bar{p}$ $\rightarrow e^{-} K_{L}^{0}$ decays to pass the trigger and offline selection requirements has been evaluated using the GEANT Monte Carlo program to generate $10^{5}$ simulated decays uniformly along the beamline within the decay tank. We find $\epsilon=(2.2 \pm 0.5)$ $\times 10^{-5}$, where the uncertainty on $\epsilon$ arises from the systematic uncertainties on the trigger calibration, calorimeter energy scale, track reconstruction efficiency, and Monte Carlo statistics. Inserting the calculated $\epsilon$ and $\sigma_{\epsilon}$ into Eqs. (3) and (5) we obtain the result $\tau_{p}^{-} / B\left(\bar{p} \rightarrow e^{-} K_{L}^{0}\right)>9 \times 10^{3}$ yr $(90 \%$ C.L.).

\section{CONCLUSIONS}

A search has been made for 11 two-body and 2 threebody decay modes of the antiproton in which there is an electron or muon in the final state. No statistically significant signal indicating $\bar{p}$ decay has been observed. Our limits on $\tau / B$ are summarized in Table I, and are significantly more stringent than the limits currently quoted by the Particle Data Group [1]. In particular, our results place the first explicit experimental limits on the muonic decay modes of the antiproton, and the first limits on the decay modes $e^{-} \gamma \gamma$ and $e^{-} \omega$. Our most stringent limit is on the decay mode $\bar{p}$ $\rightarrow e^{-} \gamma$ for which we find $\tau / B\left(\bar{p} \rightarrow e^{-} \gamma\right)>7 \times 10^{5}$ yr $(90 \%$ C.L.), which is an improvement by a factor of 400 over the prior T861 result. Noting that our limits for the simplest 
two-body decay modes are in the range $10^{4}$ to $10^{5}$ years, Eq. (1) suggests that if there is a $C P T$ violating interaction that gives rise to these decay modes, and that is described by a dimension-6 operator, the associated mass scale $m_{X}$ is greater than $\mathrm{O}\left(10^{9}\right) \mathrm{GeV} / c^{2}$. This is well beyond the reach of direct searches at high energy colliders.

\section{ACKNOWLEDGMENTS}

The APEX experiment was performed at the Fermi National Accelerator Laboratory, which is operated by Universities Research Association, under contract DE-AC02$76 \mathrm{CH} 03000$ with the U.S. Department of Energy.
[1] Particle Data Group, C. Caso et al., Eur. Phys. J. C 3, 1 (1998).

[2] R. Bluhm, V. A. Kostelecký, and N. Russell, Phys. Rev. Lett. 79, 1432 (1997).

[3] G. Gabrielse et al., Phys. Rev. Lett. 65, 1317 (1990).

[4] R. Carosi et al., Phys. Lett. B 237, 303 (1990); CPLEAR Collaboration, R. Adler et al., ibid. 364, 239 (1995).

[5] S. Geer and D. Kennedy, "A New Limit on the Antiproton Lifetime," astro-ph/9812025.

[6] Lars Bergstrom, Joakim Edsjo, and Piero Ullio, "Cosmic antiprotons as a probe for supersymmetric dark matter?," astro-ph/9902012.

[7] R. L. Golden et al., Phys. Rev. Lett. 43, 1196 (1979); E .A. Bogomolov et al., Proc. 16th ICRC, Kyoto 1, 330 (1979); Proc. 20th ICRC, Moscow 2, 72 (1987); M. Hof et al., Astrophys. J. Lett. 467, L33 (1996); J. W. Mitchell et al., Phys. Rev. Lett. 76, 3057 (1996); A. Moiseev et al., Astrophys. J. 474, 479 (1997); K. Yoshimura et al., Phys. Rev. Lett. 75, 3792 (1995); M. Boezio et al., Astrophys. J. 487, 415 (1997).
[8] S. Geer et al., Phys. Rev. Lett. 72, 1596 (1994).

[9] APEX Collaboration, S. Geer et al., "Search for Antiproton Decay at the Fermilab Antiproton Accumulator: Proposal," FERMILAB-PROPOSAL-P-868, 1992.

[10] T. Armstrong et al., Nucl. Instrum. Methods Phys. Res. A 411, 210 (1998).

[11] M. A. Hasan et al., Nucl. Instrum. Methods Phys. Res. A 295, 73 (1990).

[12] M. Hu, Ph.D. thesis, University of Nebraska, 1998.

[13] M. Hu et al., Phys. Rev. D 58, 111101 (1998).

[14] GEANT Version 3.21, R. Brun et al., CERN Program Library.

[15] R. Cousins and V. Highland, Nucl. Instrum. Methods Phys. Res. A 320, 331 (1992).

[16] S. Geer, "A method to calculate limits in the absence of a reliable background subtraction," FERMILAB-TM-2065, 1999.

[17] B. Corbin, Ph.D. thesis, University of Los Angeles, California, 1998. 